\title{
Il team degli accessi vascolari
}

\author{
Mauro Magugliani, Antonella Montani, Lucia Pozzi, Ornella Fusè, Gianlorenzo DePadova, Marzia Moretti \\ Azienda Socio Sanitaria Territoriale Ovest Milanese, Presidio Ospedaliero di Magenta, Servizio di Nefrologia e Dialisi, Milano
}

\begin{abstract}
The vascular access team
In recent years, the need for the vascular access surveillance has been growing steadily. In our dialysis department, a dedicated multidisciplinary team is active since 10 years. The Team is composed of 2 nephrologists and 5 nurses who take care of AV related surgery, AV management in the dialysis rooms, infection control, nurses education and audit. Having an AV Team allowed to monitor and efficiently control the infection rate (0.65/1000 CVC days) and contained the AV management related costs while improving patients' quality of life. Furthermore, it has been a reason for continuous professional growth. The article describes the major organization and structural and professional characteristics of our AV Team setting.
\end{abstract}

Keywords: AV team, Hemodialysis, Vascular access

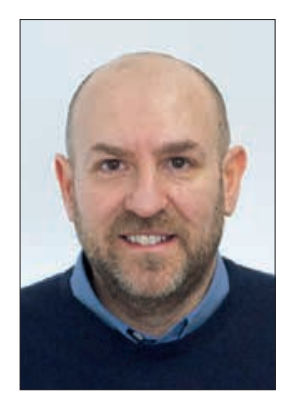

Mauro Magugliani

\section{Introduzione}

Le infezioni ospedaliere sono la complicanza più frequente e grave dell'assistenza sanitaria. I dati rilevati in Italia, nel periodo settembre/ottobre 2011, confermano la centralità del problema "infezioni correlate all'assistenza" per la sicurezza dei pazienti (1):

- su 100 pazienti ricoverati in un giorno, 6.3 presentavano un'infezione imputabile all'ambito clinico assistenziale (infezione comparsa dopo 48 ore dal ricovero in ospedale o presente al ricovero in un paziente trasferito da un altro ospedale per acuti);

- questa incidenza arriva fino al $14.8 \%$ in terapia intensiva, al $13 \%$ nei pazienti con patologia "rapidamente fatale" e, secondo McCabe (1), al 30.9\% nei pazienti intubati, al $21.4 \%$ nei pazienti portatori di catetere venoso centrale e al $13 \%$ nei pazienti portatori di catetere urinario;

Accepted: September 16, 2016

Published online: February 17, 2017

Indirizzo per la corrispondenza:

Mauro Magugliani

Servizio di Nefrologia e Dialisi

Ospedale G. Fornaroli

Via Donatore di Sangue 51

20013 Magenta (MI)

mauro.magugliani@asst-ovestmi.it
- su 100 infezioni, quelle più frequentemente riportate sono quelle respiratorie (24.1\%), a seguire ci sono le infezioni urinarie $(20.8 \%)$, le infezioni del sito chirurgico (16\%) e le batteriemie (15.8\%).

L'incremento delle infezioni ospedaliere è conseguenza della progressiva evoluzione della tecnologia nel campo assistenziale, che se da una parte permette la sopravvivenza dei pazienti ad alto rischio dall'altra consente l'ingresso dei microrganismi (anche in sedi corporee normalmente sterili), determinato dall'utilizzo di vari dispositivi quali CVC, pacemaker, protesi, cateteri vescicali e così via.

Da qui la necessità di adottare pratiche assistenziali sicure, in grado di prevenire o controllare la trasmissione delle infezioni sia in ospedale che in tutte le strutture sanitarie non ospedaliere. Occorre quindi pianificare e attuare programmi di controllo a diversi livelli (nazionale, regionale, locale). L'adozione di protocolli e procedure codificate (bundle), scritti secondo criteri di evidenza scientifica, permette di regolare e standardizzare le attività efficaci in grado di ridurre il rischio di infezioni, in primo luogo, e di indurre cambiamenti comportamentali di medici e infermieri nella gestione di dispositivi intravasali (2).

Tra i dispositivi intravasali più utilizzati per scopi diversi, vi sono i cateteri venosi centrali (CVC), che sono associati a un tasso di infezioni CVC correlate (CRBS) che va da 3.8 a 6.6 episodi/1000 giorni di permanenza per i CVC temporanei a 1.1-5.5 episodi/1000 per i CVC permanenti (3).

Uno studio condotto in un ospedale svedese, della durata di 6 anni, ha mostrato una netta riduzione dell'incidenza delle batteriemie nei pazienti portatori di CVC dopo l'istituzione di un team di sorveglianza (4). Lo studio è stato condotto in reparti di terapia intensiva e rianimazione, ma le conclusio- 
ni sono applicabili a tutti i reparti dove tali dispositivi sono utilizzati.

Nella nostra realtà di dialisi, I'istituzione del team degli AV ha dato risultati analoghi.

\section{L'evoluzione della professione infermieristica}

Nei servizi di nefrologia e dialisi, lo staff infermieristico svolge da sempre molteplici funzioni che saranno e dovranno essere ampliate considerando l'evoluzione della Professione Infermieristica, sia in termini di competenza che in termini di responsabilità (DM 739/94 relativo al Profilo Professionale, la L 42/99 che definisce l'infermieristica come professione sanitaria, il codice deontologico del 2009). Il percorso di studi universitari riconosce nuove e maggiori responsabilità agli infermieri, che in alcuni ambiti possono già muoversi in autonomia.

Negli ultimi anni molte Aziende Ospedaliere in collaborazione con le Università organizzano Master allo scopo di formare gli infermieri al posizionamento di alcuni tipi di cateteri venosi centrali a introduzione periferica (PICC) con l'utilizzo dell'ecografo. In futuro la pratica potrebbe essere estesa anche a CVC di tipo classico a inserzione giugulare, femorale o succlavia, come già avviene in altre realtà, con risultati ottimali per i pazienti (5).

È necessario, quindi, che gli infermieri siano consapevoli del ruolo che ricoprono e che siano preparati ad agire con competenza e conoscenza, intraprendendo percorsi di aggiornamento quando siano richieste ulteriori conoscenze.

Alcuni studi $(6,7)$ documentano che infermieri con un livello di formazione avanzato migliorano gli esiti sui pazienti. La relazione tra qualità e quantità degli infermieri rispetto agli esiti sui pazienti nelle aree di assistenza ospedaliera sia intensiva che sub intensiva è ampiamente documentata e riconducibile anche alle situazioni di cronicità e di acuzie tipiche dell'ambito nefrologico specifico.

\section{La nostra esperienza}

Presso il nostro servizio di nefrologia e dialisi, lo staff infermieristico è andato acquisendo da anni sempre più competenze nel proprio lavoro integrandole con quelle dello staff medico.

Gli infermieri, oltre alla preparazione dei reni artificiali (sempre più sofisticati) e alla conduzione pura della seduta dialitica, si occupano infatti dell'educazione e della gestione dell'accesso vascolare, dell'educazione alimentare e dell'interazione con la figura medica nell'ambulatorio di pre-dialisi e trapianto.

L'infermiere di dialisi ha la responsabilità di gestire in completa autonomia gli attacchi di FAV e di CVC, monitorizzandoli nel tempo al fine di ridurne le complicanze infettive e ostruttive e di conseguenza prolungandone la sopravvivenza.

Dal 2003 è inoltre attivo un team che si occupa in modo specifico della sorveglianza degli accessi vascolari, siano essi CVC o FAV.

\section{Il team degli accessi vascolari}

Lo scopo del team degli accessi vascolari è quello di sorvegliare gli Accessi Vascolari (AV), attraverso la stesura di protocolli e procedure basati su evidenze scientifiche uniformando gli interventi e i comportamenti per migliorarne la gestione, ridurne le complicanze e migliorare l'efficienza e la cura del paziente in emodialisi. Il team degli AV è una realtà ben radicata nel nostro servizio di emodialisi, è composto da un medico nefrologo referente, da un medico che collabora agli impianti di CVC (cateteri venosi centrali) e all'allestimento di FAV (fistole artero-venose) e da un numero di infermieri $(6,7)$ che supportano tutta l'attività medica e chirurgica relativa agli $\mathrm{AV}$.

La formazione infermieristica viene eseguita sul campo e indispensabili sono 1) la disponibilità dei soggetti di farsi carico di un compito aggiuntivo per migliorare la qualità assistenziale e 2) i momenti di verifica (audit) interni che il gruppo svolge con regolarità (ogni 3 mesi e ogni volta che si presenta un problema), al fine di valutare il proprio operato e di proporre eventuali miglioramenti.

Non ci sono criteri specifici per entrare a far parte del team: chi vuole dedicarsi in maniera più specifica e approfondita agli $\mathrm{AV}$, occupandosene direttamente (dal processo decisionale all'impianto e fino alla gestione), per interesse e motivazioni proprie, può chiedere di far parte inizialmente del pool di sala operatoria. II gruppo di sala si occupa di seguire tutti gli AV siano essi CVC o FAV. Con il tempo, per esperienza e per conoscenza e continuo aggiornamento, alcuni si occupano in maniera più specifica di fistole (p. es., prime punture e controllo nel tempo per complicazioni varie, buttonhole, ecc.). I dati di monitoraggio vengono raccolti dagli infermieri di sala dialisi. Sui dati raccolti vengono effettuati gli audit per modificare comportamenti o azioni non corretti di tutto il personale.

II nostro team viene facilmente contattato da tutto il personale infermieristico del nostro Ospedale in presenza di un problema specifico su un accesso vascolare. A volte le consulenze sono svolte direttamente nel servizio che ne fa richiesta. I nostri pazienti, ricoverati o non, sono istruiti per contattarci il prima possibile su qualsiasi dubbio abbiano circa la loro FAV o il loro CVC.

\section{Caratteristiche strutturali, logistiche e organizzative}

La struttura di dialisi gestisce le attività nefrologiche e dialitiche dell'ospedale di Magenta a cui afferisce anche un centro dialisi ad assistenza limitata (CAL). L'attività nefrologica si svolge su 4 letti di degenza e quella dialitica su 16 posti tecnici, distribuiti su 5 stanze di emodialisi ospedaliera e su 8 posti dialisi al CAL. La Struttura esegue inoltre l'attività di dialisi pe- 
ritoneale e l'attività ambulatoriale di primo e secondo livello.

Il reparto di dialisi si sviluppa attorno a un corridoio dove da un lato si trovano le stanze per il trattamento emodialitico e dall'altro sono ubicati gli studi (medici, coordinatore infermieristico), stanze utilizzate come deposito per l'enorme quantità di materiale monouso e spogliatoi per i pazienti (maschile e femminile). Inoltre sono presenti 2 stanze utilizzate per la dialisi peritoneale e un'altra stanza per il dayhospital, un laboratorio adibito a officina per la riparazione e disinfezione delle apparecchiature e i locali dell'impianto di trattamento di osmosi dell'acqua. Nello stesso corridoio si affacciano due locali adibiti a sala operatoria per interventi di allestimento FAV o posizionamenti di CVC. Sala ad uso esclusivo del reparto di dialisi

L'attività di sala operatoria richiede la disponibilità di un gruppo di infermieri (sempre presenti in turno) che a rotazione si occupano di seguire gli interventi di allestimento FAV e del posizionamento di CVC. L'attività di sala operatoria può essere programmata parzialmente con le fistole e con CVC definitivi. Al contrario, il posizionamento di CVC temporanei avviene in urgenza.

In ogni stanza di dialisi è presente un infermiere e in ogni turno è previsto un infermiere "fuori" dalle stanze di dialisi (jolly), che si occupa del lavoro di supporto all'assistenza (attacchi, stacchi, esami ematici, controllo carrello urgenza, attività amministrative, urgenze dialitiche in altri reparti ecc.). Se l'infermiere jolly non fa parte dello staff di sala, avvicenda momentaneamente l'infermiere del gruppo di sala operatoria per l'attività in sala dialisi, sostituendolo per il tempo necessario per l'attività urgente di sala operatoria. Questo può avvenire poiché la stanza dedicata alla sala operatoria è integrata nella struttura logistica della UO. Spesso l'infermiere di giornata viene assegnato come infermiere jolly ma, se l'attività operatoria è programmata, al mattino viene prevista un'unità infermieristica supplementare.

\section{Competenze degli infermieri del team}

\section{In Sala Operatoria}

In sala operatoria l'infermiere accoglie il paziente e controlla gli esami pre intervento (check list) e la corretta compilazione dei moduli di consenso informato, prepara il paziente con la detersione e la disinfezione della sede dell'intervento, posiziona un catetere venoso periferico, monitorizza i parametri vitali e, quando richiesto, posiziona la piastra per elettrobisturi. Inoltre, collabora nella vestizione del medico nefrologo e nella preparazione del campo operatorio secondo i protocolli in uso in reparto.

Durante il posizionamento del CVC controlla il tracciato dell'ECG per verificare eventuali alterazioni del ritmo cardiaco causate dalla guida metallica del catetere, posiziona la sonda ecografica per consentire al medico l'introduzione eco guidata ed è di supporto durante il posizionamento del CVC.
Al termine del posizionamento provvede a eseguire un ECG endocavitario in collaborazione con il medico impiantatore per controllare la posizione del dispositivo. Esegue la medicazione finale e si accerta che il paziente esegua una RX TORACE di controllo.

Durante l'allestimento della FAV l'infermiere controlla periodicamente i parametri vitali del paziente e si occupa di gestire la sala durante l'intervento.

Al paziente viene consegnata una nota informativa sulla gestione della FAV e sul controllo del suo buon funzionamento. L'infermiere ne verifica la comprensione, risponde a eventuali chiarimenti e istruisce sulla pratica da effettuare nel post operatorio (al paziente viene consegnata una pallina antistress per gli esercizi indicati).

Il controllo delle giacenze e delle scadenze del materiale della sala operatoria è affidato con cadenza settimanale al personale del team.

\section{Nelle sale dialisi}

All'interno del servizio di dialisi, al team è affidata la sorveglianza dei protocolli di gestione dei CVC e delle FAV, per le fasi di medicazione dei CVC e di attacco e stacco. Grazie a un continuo lavoro di aggiornamento e confronto tra i membri del team vascolare, l'infermiere acquisisce competenza per giudicare l'eventuale presenza di infezioni dell'exit site o del tunnel del CVC e valuta se la situazione richieda una valutazione del nefrologo dedicato o se rientra nelle proprie competenze. È inoltre chiamato dai colleghi come consulente, in presenza di problematiche o dubbi circa gli AV. Verifica la corretta compilazione delle schede di controllo dei CVC e delle FAV e, se sono presenti problematiche ricorrenti, segnala le situazioni che richiedono un approfondimento clinico.

Al team viene affidato il monitoraggio delle FAV per valutare la presenza di eventuali stenosi tramite l'utilizzo del $Q B$ / StressTest (o test di Bonforte)(8); in presenza di test positivo è contattato il nefrologo referente che, dopo aver effettuato anch'egli l'esame obiettivo e l'ecocolordoppler, identifica le problematiche e pianifica le procedure specifiche per una rapida risoluzione del problema, riducendo, così, il rischio di chiusura definitiva della FAV.

Alcuni infermieri del team hanno un'elevata manualità nella venopuntura e, quindi, vengono chiamati per l'inserzione degli aghi con FAV di recente allestimento o qualora si verifichino problematiche. Inoltre, nell'ultimo anno è stato dato loro l'incarico di iniziare la tecnica buttonhole in alcuni pazienti appositamente selezionati.

Essendo la nostra Azienda articolata in 4 presidi (2 CAL e 2 Centri Madre) nell'ultimo anno, si stanno predisponendo dei piani di interscambio tra le diverse realtà per uniformare i criteri di gestione degli AV, utilizzando l'esperienza maturata dalla nostra equipe. 


\section{Discussione}

Nonostante la nascita di questo gruppo sia stata vista in modo negativo da una parte dello staff medico-infermieristico, perché si riteneva potesse togliere la professionalità altrui o interferire con essa, con il tempo tutti ne hanno compreso I'importanza e lo hanno accettato, anzi la presenza di questo team ha stimolato la creazione di altri gruppi di competenza specifica: gestione degli esami di routine, esami settimanali, gestione pazienti in lista trapianto, gestione dell'ambulatorio di pre dialisi e così via.

La presenza di un team di sorveglianza degli accessi vascolari, da oltre 10 anni, nel nostro servizio di nefrologia e dialisi, ha permesso di ottenere ottimi risultati nel controllo delle infezioni CVC, stabili da anni a 0.65 per 1000 giorni di permanenza (9), che corrisponde al grado di eccellenza secondo la tabella di Beathard (10). Gli accessi vascolari nativi e/o protesici hanno una sopravvivenza del $62 \%$ a 3 anni, con un conseguente miglioramento della qualità di vita del nostro paziente e una riduzione dei costi di "gestione" del paziente stesso.

Fondamentali sono stati: il supporto ricevuto dal coordinatore infermieristico, la collaborazione degli infermieri che hanno creduto nel progetto e ne hanno dato l'avvio e la fiducia e la disponibilità del direttore medico e del nefrologo referente per gli AV che hanno saputo motivare e sostenere tale scelta nel tempo.

\section{Conclusioni}

Possiamo affermare che la presenza di questo team continua a contribuire a migliorare la qualità assistenziale con abbattimento dei rischi di infezione e trombosi degli AV.

Il team ha creato uno stimolo culturale che spinge a un continuo aggiornamento e a una crescita professionale all'interno sia del team che di tutto il personale, contribuendo a ridurre il turn-over, a diminuire il rischio di burnout e a migliorare la percezione professionale sia all'interno che all'esterno del servizio.

Non esiste al momento, nella nostra azienda, una posizione funzionale specifica per i membri del team degli AV.

Sarebbe inoltre auspicabile la creazione di uno strumento elettronico (cartella/scheda) unificato che possa contribuire a creare evidenze e a facilitare la ricerca clinica. Inoltre, riteniamo che la presenza di dirigenti e coordinatori propositivi sia indispensabile per favorire la crescita e lo sviluppo di equipe, o mini-equipe con specifiche e proprie responsabilità.

Il livello di preparazione della professione infermieristi- ca aumenta costantemente e parallelamente le competenze espresse, che sono sempre più una realtà, una necessità e un valore aggiunto per il SSN e per il cittadino.

\section{Ringraziamenti}

Si ringraziano il Direttore di Dipartimento Dottor Carlo Guastoni e il nefrologo referente per gli accessi vascolari Dottoressa Marina Cornacchiari, per aver creduto e averci supportato in questo progetto e Marisa Pegoraro, referente editoriale della Filiale Italiana EDTNA/ERCA per la revisione del manoscritto.

\section{Disclosures}

Financial support: No financial support was received for this submission.

Conflict of interest: The authors have no conflict of interest.

\section{Bibliografia}

1. http:/www.epicentro.iss.it./problemi/infezioni_correlate/IcaReportEcdc2013.asp - II portale dell'epidemiologia per la sanità pubblica. Accesso 15 giugno, 2016.

2. Jackson $A$. Reflecting on the nursing contribution to vascular access. Br J Nurs. 2003;12(11):657-665.

3. Cornacchiari M. Prevenzione delle infezioni catetere venoso centrale correlate (CRBSI). Giornale di Tecniche Nefrologiche e Dialitiche. 2013;25(3):220-224.

4. Hammarskjöld F, Berg S, Hanberger H, Taxbro K, Malmvall BE. Sustained low incidence of central venous catheter-related infections over six years in a Swedish hospital with an active central venous catheter team. Am J Infect Control. 2014;42(2):122-128.

5. Yacopetti N, Alexandrou E, Spencer TR, et al. Central venous catheter insertion by a clinical nurse consultant or anaesthetic medical staff: a single-centre observational study. Crit Care Resusc. 2010;12(2):90-95.

6. Rafferty AM, Clarke SP, Coles J, et al. Outcomes of variation in hospital nurse staffing in English hospitals: cross-sectional analysis of survey data and discharge records. Int J Nurs Stud. 2007;44(2):175-182.

7. Aiken LH, Sloane DM, Bruyneel L, et al. Nurse staffing and education and hospital mortality in nine European countries: a retrospective observational study. Lancet. 2014;383(9931): 1824-1830.

8. Bonforte G, Pogliani D, Brenna S, et al. Validation of QB stress test as a useful tool in the detection of native arteriovenous fistula stenosis: results after 22 months of follow-up. Nephrol Dial Transplant. 2010;25(6):1943-1949.

9. Cornacchiari M, Heidempergher M, Stasi A, et al. Effectiveness of a protocol for the prevention of hemodialysis venous catheter-related infections. J Vasc Access. 2011;12(4): 313-317.

10. Beathard GA, Urbanes A. Infection associated with tunneled hemodialysis catheters. Sem Dial. 2008;21:528-538. 